Pacific Journal of Mathematics

ON THE EXPANSION IN JOINT GENERALIZED

KLAUS Kalb 


\title{
ON THE EXPANSION IN JOINT GENERALIZED EIGENVECTORS
}

\author{
KLAUS Gero KaLB
}

\begin{abstract}
Let $\mathscr{C}$ be a family commuting selfadjoint of (normal) operators in a complex (not necessarily separable) Hilbert space $H$. A natural triplet $\phi \subset H \subset \phi^{\prime}$ is described, such that (1) $\mathscr{A}$ possesses a complete system of joint generalized eigenvectors in $\phi^{\prime}$; (2) the joint generalized point spectrum of $\mathscr{A}$ essentially coincides with the joint spectrum of $\mathscr{A}$; (3) the generalized point spectra, generalized spectra and spectra essentially coincide for all $A \in \mathscr{A}$; (4) the simultaneous diagonalization of $\mathscr{A}$ in $H$ by means of its spectral measure extends to $\phi^{\prime}$. Also the multiplicity of the joint generalized eigenvectors of $\mathscr{A}$ is discussed.
\end{abstract}

Let $\phi$ be a locally convex space, which is embedded densely and continiously into $H$, such that $A \phi \subset \phi$ and $\dot{A}=A \mid \phi \in \mathscr{L}(\phi)$ for all $A \epsilon$ A. Consider the triplet $\phi \subset H \subset \phi^{\prime}$. A joint generalized eigenvector of $\mathscr{A}$ with respect to the joint generalized eigenvalue $\left(\lambda_{A}\right)_{A \in \mathscr{A}} \in$ $\Pi_{A \in \mathscr{Y}} C$ is a continuous linear form $x^{\prime} \in \phi^{\prime}$ such that

$$
x^{\prime} \neq 0 \text { and } \dot{A}^{\prime} x^{\prime}=\lambda_{A} \cdot x^{\prime} \text { for all } A \in \mathscr{A} .
$$

The system $\&$ of all joint generalized eigenvectors of $\mathscr{A}$ is called complete, if $\left\langle\varphi, e^{\prime}\right\rangle=0$ for all $e^{\prime} \in \mathbb{E}$ implies $\phi=0(\phi \in \phi)$. For $H$ separable there is a number of conditions on $\phi$, under which $₹$ is complete (cf. e.g., [14], [3]), and there also are effective constructions of $\phi$ with respect to a given family $\mathscr{A}$ (cf. [13], [14] for $\mathscr{A}$ countable; [15]). The fact that especially in the case of a single normal operator there generally exist many more joint generalized eigenvalues and eigennvectors than necessary (and reasonable in physical applications) has led to recent investigations ([15], [16]; [1]; [2]; [5]; [8], [9]). Let $\sigma_{P}\left(\mathscr{A}^{\prime}\right)$ be the joint generalized point spectrum of $\mathscr{A}$ (i.e., the set of all joint generalized eigenvalues of $\mathscr{A}$ ), let $\sigma(\mathscr{A})$ be the joint spectrum of $\mathscr{A}$ as defined in Gelfand theory (cf. $\S 2$ ). Let $\mathscr{B}$ be the (commutative) $C^{*}$-algebra generated by $\mathscr{A}$ and 1 . In the present work we propose the construction of a natural triplet $\phi \subset$ $H \subset \phi^{\prime}$, by which the following is achieved:

(a) $\sigma_{P}\left(\mathscr{\mathscr { A }}^{\prime}\right) \subset \overline{\sigma_{P}\left(\mathscr{\mathscr { C } ^ { \prime }}\right)}=\sigma(\mathscr{A})$;

(b) $\sigma_{P}\left(\dot{B}^{\prime}\right) \subset \overline{\sigma_{P}\left(\dot{B}^{\prime}\right)}=\sigma\left(\dot{B}^{\prime}\right)=\sigma(B)$ for all $B \in \mathscr{B}$;

(c) the simultaneous diagonalization of $\mathscr{B}$ by means of its spectral measure can be transferred to $\dot{\mathscr{B}}^{\prime}$. 
For $H$ separable we can even attain $\sigma_{P}\left(\mathscr{A}^{\prime}\right)=\sigma(\mathscr{L})$ and $\sigma_{P}\left(\dot{B}^{\prime}\right)=$ $\sigma(B)$ for all $B \in \mathscr{B}$, and also have a description of the multiplicity of the joint generalized eigenvalues.

In the case of a single selfadjoint operator our method reduces to that of [9] (cf. also [11]) and for $\mathscr{A}=\mathscr{B}$ is similar to that of [15] where for $H$ separable the equation $\sigma(\mathscr{B})=\sigma_{P}\left(\mathscr{\mathscr { B }}^{\prime}\right)$ is realized. The basic idea of the construction, due to R. A. Hirschfeld [7], is to choose (by means of an appropriate spectral representation of $\mathscr{B}$ ) the space $\phi$ as a space of continuous functions with compact support on a locally compact space $R$ (or as a space of continuous vector fields, if the theory of R. Godement [6] is used), such that the joint generalized eigenvectors essentially are the point masses (characters).

2. Simultaneous diagonalization and spectral decomposition. In this section we summarize the spectral and multiplicity theory of [17], [18], [19]. Let $S$ be the spectrum of $\mathscr{B}$, i.e., the set of all (continuous) homomorphisms of $\mathscr{B}$ onto $C$, endowed with the usual topology. Let $\hat{B}(\cdot): S \rightarrow C$, defined by $\hat{B}(s)=s(B)(s \in S)$, be the Gelfand transform of $B \in \mathscr{B}$. The application $\mathscr{B} \ni B \mapsto \widehat{B}(\cdot) \in$ $C(S)$ is an isometrical $*$-isomorphism of $\mathscr{B}$ onto $C(S)$. Let $E(\cdot)$ be the spectral measure of $\mathscr{B}: B=\int_{S} \hat{B}(s) d E(s)(B \in \mathscr{B})$. The joint spectrum (cf. [18], p. 150) of $\mathscr{A}$, denoted $\sigma(\mathscr{A})$ ), is defined by $\sigma(\mathscr{A})=$ $\left\{(\hat{A}(s))_{A \in \mathscr{A}}: s \in S\right\} . \quad \sigma(\mathscr{A}) \subset \Pi_{A \in}, \sigma(A)$ is homeomorphic to $S$ under the application

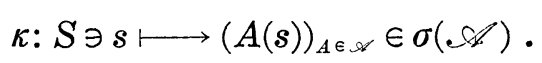

Choose a decomposition $H=\bigoplus_{i \in I} H_{i}$, such that $\mathscr{B} H_{i} \subset H_{i}$ and $\mathscr{B}_{i}=$ $\left.\mathscr{B}\right|_{H_{i}}$ possesses a cyclic vector $x_{i}(i \in I)$. Let $S_{i}$ be the spectrum of $\mathscr{B}_{i}(i \in I)$. Then there is a family $\left(m_{i}\right)_{i \in I}$ of positive Borel measures on $S_{i}$ with support $S_{i}$ inducing a spectral representation $H \leftrightarrow$ $\bigoplus_{i \in I} L^{2}\left(S_{i}, m_{i}\right)$. Thereby $H_{i}$ is transferred in $L^{2}\left(S_{i}, m_{i}\right)$, especially $x_{i}$ in $1_{S_{i}}(i \in I)$; an operator $B \in \mathscr{B}$ is converted in the multiplication by $\left(\hat{B}_{i}(\cdot)\right)_{i \in I}$, where $\hat{B}_{i}(\cdot)\left(=\left.\hat{B}(\cdot)\right|_{S_{i}}\right.$ if $S_{i}$ is considered as a subset of $S)$ denotes the Gelfand transform of $\left.B\right|_{H_{i}}(i \in I)$; a spectral projection $E(\mathfrak{b}), \mathfrak{b}$ a Borel subset of $S$, is transferred in the multiplication by $\left(\chi_{5 \cap s_{i}}\right)_{i \in I}$. Finally we have $m_{i}(\cdot)=\left(E(\cdot) x_{i}, x_{i}\right)(i \in I)$. When $H$ is separable, we can choose $I=N$ and achieve by a normalization (cf. [17], [10]) that (in an essentially unique manner) $m_{1}>m_{2}>\cdots$, particularly $S=S_{1} \supset S_{2} \supset \cdots$. The (well defined) function

$$
m_{H}(s)=\sharp\left\{n \in N: s \in S_{n}\right\} \quad(s \in S)
$$


is called the Hellinger-Hahn multiplicity function of $\mathscr{B}$.

We return to the general case, in which, for the sake of simplification of notation, we formulate the affirmations concerning spectral decompositions in a somewhat different way (cf. [19]): We consider the sets $S_{i}(i \in I)$ as pairwise disjoint sets $\widetilde{S}_{i}(i \in I)$ and define $R=\cup_{i \in I} \widetilde{S}_{i}$. A set $V \subset R$ is defined to be open, if for all $i \in I$ the set $V \cap \widetilde{S}_{i}$ (interpreted as a subset of $S_{i}$ ) is open in $S_{i}$. With that $R$ is a locally compact topological Hausdorff space; each $S_{i}$ is open and compact in $R$. A function $f: R \rightarrow C$ belongs to $C_{c}(R)$ if and only if $\left.f\right|_{\tilde{s}_{i}} \in C\left(S_{i}\right)$ for all $i \in I$ and $\left.f\right|_{\bar{s}_{i}}=0$ for all but finitely many $i \in I$. Define a Radon measure $\mu$ on $R$ by

$$
\mu(f)=\int_{R} f \cdot d \mu=\sum_{i \in I} \int_{S_{i}} f \cdot d m_{i} \quad\left(f \in C_{c}(R)\right) .
$$

Then there is a spectral representation $H \leftrightarrow L^{2}(R, \mu)$ of $\mathscr{B}$ by which $\mathscr{B}$ is converted in a subalgebra of the multiplication algebra $B C(R)$ (:=algebra of bounded continuous numerical functions on $R$ ) on $L^{2}(R, \mu): \mathscr{B} \ni B \mapsto$ multiplication by $\widetilde{B}(\cdot) \in B C(R)$, where $\widetilde{B}(r):=\widehat{B}(\lambda r)$ $(r \in R)$. Here $\lambda: R \rightarrow \bigcup_{i \in I} S_{i} \subset S$ is the natural surjection. Finally we shall need:

$$
E(\cdot) \text { is concentrated on } \bigcup_{i \in I} S_{i} \text {; particularly } \overline{\bigcup_{i \in I} S_{i}}=S \text {; }
$$

$$
\begin{gathered}
\|B\|=|\hat{B}(\cdot)|_{C(S)}=|\widetilde{B}(\cdot)|_{B C(R)} \quad(B \in \mathscr{B}) ; \\
\sigma(B)=\hat{B}(S)=\overline{\widetilde{B}(R)} \quad(B \in \mathscr{B}) .
\end{gathered}
$$

$(|\cdot|$ denotes the supremum norm.)

3. Expansion in joint generalized eigenvectors. We proceed now to the construction of the triplet $\phi \subset H \subset \phi^{\prime}$. We assume without loss of generality that $H=L^{2}(R, \mu) \leftrightarrow \bigoplus_{i \in I} L^{2}\left(S_{i}, m_{i}\right)$ and $\mathscr{B} \subset$ $C B(R)$. Let $\phi:=C_{c}(R)$. It is easy to see that $\phi$ is topologically isomorphic to the locally convex direct sum $\dot{\sum}_{i \in I} C\left(S_{i}\right)$ (considered in [9]). $\phi$ satisfies with respect to $\mathscr{B}$ (and $\mathscr{A}$ ) all the prerequisites listed in the introduction. For $r \in R$ define $e^{\prime}(r) \in \phi^{\prime}$ by $\left\langle\varphi, e^{\prime}(r)\right\rangle=$ $\varphi(r)(\varphi \in \phi)$.

Theorem (3.1). (i ) $\dot{B}^{\prime} e^{\prime}(r)=\widetilde{B}(r) \cdot e^{\prime}(r)(B \in \mathscr{B}, r \in R)$.

(ii) $(\varphi, \psi)=\int_{R}\left\langle\varphi, e^{\prime}(r)\right\rangle \overline{\left\langle\psi, e^{\prime}(r)\right\rangle} d \mu(r)(\varphi, \psi \in \phi)$ [(i) and (ii) mean that $\quad \mathcal{F}=\left\{e^{\prime}(r): r \in R\right\}$ is a complete system of joint generalized eigenvectors of $\mathscr{B}]$.

(iii) $\sigma_{P}\left(\dot{B}^{\prime}\right)=\widetilde{B}(R)(B \in \mathscr{B})$. 
(iv) $\sigma\left(\dot{B}^{\prime}\right)=\overline{\sigma_{c 1}\left(\dot{B}^{\prime}\right)}=\sigma(B)(B \in \mathscr{B})$.

Here $\sigma\left(\dot{B}^{\prime}\right)$ denotes the spectrum of $\dot{B}^{\prime}$ in the sense of Waelbroeck (cf. e.g., [12]) and $\sigma_{c 1}\left(\dot{B}^{\prime}\right)$ is defined as the set of those $z \in C$, for which $\dot{B}^{\prime}-z$ is not invertible in $\mathscr{L}\left(\phi^{\prime}\right)$. Thereby on $\phi^{\prime}$ always is considered the strong topology and on $\mathscr{L}\left(\phi^{\prime}\right)$ the topology of uniform convergence on bounded subsets of $\phi$.

Proof. (i), (ii) are direct consequences of our construction. (iii): Let $B \in \mathscr{B}$. Because of (i) we only have to show that $\sigma_{P}\left(\dot{B}^{\prime}\right) \subset \widetilde{B}(R)$. Let $z \in \sigma_{P}\left(\dot{B}^{\prime}\right)$ and suppose that $z \notin \widetilde{B}(R)$. Choose $x^{\prime} \in \phi^{\prime}$ such that $x^{\prime} \neq 0$ and $\dot{B}^{\prime} x^{\prime}=z x^{\prime}$. Let $\varphi \in \phi$ be arbitrary. Then there exists $\psi \in \phi$ such that $\varphi(r)=(\widetilde{B}(r)-z) \cdot \psi(r)(r \in R)$. Hence $\left\langle\varphi, x^{\prime}\right\rangle=\langle(\widetilde{B}(\cdot)-z)$. $\left.\psi(\cdot), x^{\prime}\right\rangle=\left\langle\psi,\left(\dot{B}^{\prime}-z\right) x^{\prime}\right\rangle=0$, i.e., $x^{\prime}=0$. Contradiction. (iv): By (iii) we have $\sigma(B)=\overline{\widetilde{B}(R)}=\overline{\sigma_{P}\left(\dot{B}^{\prime}\right)} \subset \overline{\sigma_{c 1}\left(\dot{B}^{\prime}\right)} \subset \sigma\left(\dot{B}^{\prime}\right)$. It remains to show that $\sigma\left(\dot{B}^{\prime}\right) \subset \overline{\widetilde{B}(R)}$ : Let $z \notin \overline{\widetilde{B}(R)}$. To demonstrate that $z \notin \sigma\left(\dot{B}^{\prime}\right)$, the two cases $z=\infty$ and $z \in C$ have to be treated seperately. Let $z=\infty$. Choose $C>0$ such that $|\widetilde{B}(r)| \leqq C(r \in R)$. Then $U:=\{\infty\} \cup$ $\{w \in C:|w| \geqq 2 \cdot C\}$ is a neighborhood of $\infty$, and $\left|(\widetilde{B}(r)-w)^{-1}\right| \leqq 1 / C$ $(r \in R)$ for $w \in U \cap C$. For $w \in U \cap C$ define $Q(w) \in \mathscr{L}\left(\phi^{\prime}\right)$ by

$$
\left\langle\varphi, Q(w) x^{\prime}\right\rangle=\left\langle(\widetilde{B}(\cdot)-w)^{-1} \cdot \phi(\cdot), x^{\prime}\right\rangle \quad\left(\varphi \in \phi, x^{\prime} \in \phi^{\prime}\right) .
$$

It is clear that $Q(w)\left(\dot{B}^{\prime}-w\right)=\left(\dot{B}^{\prime}-w\right) Q(w)=1$ for all $w \in U \cap C$ and easy to see that $\{Q(w): w \in U \cap C\}$ is bounded in $\mathscr{L}\left(\phi^{\prime}\right)$. Hence $\infty \notin \sigma\left(\dot{B}^{\prime}\right)$. If $z \in C$, choose a neighbourhood $V$ of $z$ such that $\bar{V} \cap$ $\overline{\widetilde{B}(R)}=\varnothing$ and proceed similarity.

We shall show now that the spectral measure $E(\cdot)$ of $\mathscr{B}$ can be extended to a spectral measure of $\mathscr{\mathscr { B }}^{\prime}$.

THeOREM (3.2). There is an (unique) spectral measure $P(\cdot)$ on $S$ with values in $\mathscr{L}\left(\phi^{\prime}\right)$ such that $\dot{B}^{\prime}=\int_{S} \hat{B}(s) \cdot d P(s)(B \in \mathscr{B})$ and $\left.P(\cdot)\right|_{H}=E(\cdot)$.

Proof. $\quad \phi^{\prime}$ is the space of Radon measures on $R$. Define $P(\mathfrak{b}) x^{\prime}=$ $\chi_{\lambda^{-1(\mathfrak{b})}} \cdot x^{\prime}\left(\mathfrak{b}\right.$ a Borel subset of $\left.S, x^{\prime} \in \phi^{\prime}\right)$, i.e., $\left\langle\varphi, P(\mathfrak{b}) x^{\prime}\right\rangle=\int_{\lambda^{-1(\mathfrak{b})}} \varphi \cdot d x^{\prime}$ for $\varphi \in \phi$. It is easily chequed that $P(\cdot)$ is a bounded $\sigma$-additive spectral measure in $\mathscr{L}\left(\phi^{\prime}\right)$ and that $\left.P(\cdot)\right|_{H}=E(\cdot)$. Since $\phi^{\prime}$ is complete and barrelled, the integral $\int_{S} \hat{B}(s) \cdot d P(s)(B \in \mathscr{B})$ exists in the 
strong sense. An easy calculation shows that $\left\langle\varphi, \int_{S} \hat{B}(s) \cdot d P(s) x^{\prime}\right\rangle=$ $\int_{S} \hat{B}(s) d\left\langle\varphi, P(s) x^{\prime}\right\rangle=\left\langle B \phi, x^{\prime}\right\rangle$ for all $\varphi \in \phi, x^{\prime} \in \phi^{\prime}$, i.e., $\int_{S} \hat{B}(s) \cdot d P(s)=\dot{B}^{\prime}$.

We now discuss the relations between the joint spectrum and the joint generalized point spectrum of $\mathscr{A}$ :

THEOREM (3.3). $\quad \sigma_{P}\left(\mathscr{\mathscr { A }}^{\prime}\right) \subset \overline{\sigma_{P}\left(\dot{\mathscr{A}}^{\prime}\right)}=\sigma(\mathscr{A})$.

Proof. For $r \in R$ we have by Theorem (3.1) (i) that $(\tilde{A}(r))_{A \in:}=$ $(\hat{A}(\lambda r))_{A \in \mathscr{C}} \in \sigma_{P}\left(\dot{\mathscr{A}}^{\prime}\right) \quad(r \in R)$. Hence $\kappa(\lambda(R))=\kappa\left(\mathrm{U}_{i \in I} S_{i}\right) \subset \sigma_{P}\left(\dot{\mathscr{A}}^{\prime}\right)$, where $\kappa$ is the homeomorphism of (2.1). Because of (2.3) we obtain

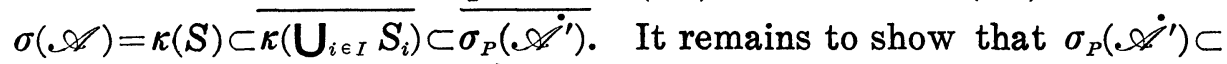
$\sigma(\mathscr{A})$. Let $\left(\lambda_{A}\right)_{A \in \mathscr{r}} \in \sigma_{P}\left(\mathscr{A}^{\prime}\right)$; let $x^{\prime} \in \phi^{\prime}=C_{c}^{\prime}(R)$ be a joint generalized eigenvector of $\mathscr{A}$, i.e., (1.1) holds. Choose $i \in I$ such that $x_{i}^{\prime}=\left.x^{\prime}\right|_{C\left(s_{i}\right)} \neq 0$. Consider the triplet $\phi_{i} \subset H \subset \phi_{i}^{\prime}$, where $\phi_{i}=C\left(S_{i}\right), H=L^{2}\left(S_{i}, m_{i}\right)$. We then have $\left(\left.A\right|_{\phi_{i}}\right)^{\prime} x_{i}^{\prime}=\lambda_{A} \cdot x_{i}^{\prime}(A \in \mathscr{A})$. We shall show that there exists an (unique) $s_{i} \in S_{i}$, such that $\lambda_{A}=\hat{A}\left(s_{i}\right) \quad(A \in \mathscr{A})$. For the sake of simplification of notation we suppress the index $i$, i.e., we consider the case of total multiplicity 1 without loss of generality. We first extend the function

$$
\text { . } \ni A \longmapsto \lambda_{A} \in C
$$

to $\mathscr{B}$ such that (1.1) remains valid. To do this, let $\mathscr{P}(\mathscr{A})$ be the algebra of polynomials in elements of $\mathscr{A}$ and 1 . The closure of $\mathscr{P}(\mathscr{A})$ in $\mathscr{L}(H)$ equals $\mathscr{B}$. If $p=p\left(\alpha_{1}, \cdots, \alpha_{n}\right)$ is a polynomial in $n$ variables, we define $\lambda_{B}=p\left(\lambda_{A_{1}}, \cdots, \lambda_{A_{n}}\right)$ for $B=p\left(A_{1}, \cdots, A_{n}\right) \epsilon$ $\mathscr{P}(\mathscr{A})$. By (1.1) we conclude that the function

$$
\mathscr{P}(\mathscr{L}) \ni B \longmapsto \lambda_{B} \in C
$$

is well defined, constitutes an extension of (3.4) and satisfies

$$
\dot{B}^{\prime} x^{\prime}=\lambda_{B} \cdot x^{\prime} \quad(B \in \mathscr{P}(\mathscr{A})) \text {. }
$$

Observing that $\lambda_{B} \in \sigma_{P}\left(\dot{B}^{\prime}\right) \subset \sigma(B)$ (cf. (3.1) (iii), hence $\left|\lambda_{B}\right| \leqq\|B\|$, we obtain that the (linear) function (3.5) is continuous. Hence it possesses an unique extension as a continuous function on $\mathscr{B}$, which we again denote by $B \mapsto \lambda_{B}$ and which satisfies for reasons of continuity the relations

$$
\dot{B}^{\prime} x^{\prime}=\lambda_{B} \cdot x^{\prime} \quad(B \in \mathscr{B}) .
$$

Using this it is easily chequed that $B \mapsto \lambda_{B}$ is an homomorphism of $\mathscr{B}$ onto $C$ (cf. [15]), i.e., defines an element $s \in S$ such that $\lambda_{B}=$ $s(B)=\hat{B}(s)(B \in \mathscr{B})$. 
The proof shows particularly that a joint generalized eigenvector of $\mathscr{A}$ is automatically one of $\mathscr{B}$.

4. The multiplicity of the joint generalized eigenvalues. First we give a supplement to the second part of the proof of Theorem (3.3):

LEMMA (4.1). $x^{\prime}$ is a multiple of point mass in $s$.

Proof. Recall that $R=S$ (according to our reduction to the cyclic case). (3.7) then means that

$$
\left\langle\hat{B}(\cdot) \cdot \varphi(\cdot), x^{\prime}\right\rangle=\hat{B}(s) \cdot\left\langle\varphi, x^{\prime}\right\rangle \quad(\varphi \in C(S), \hat{B}(\cdot) \in C(S)) .
$$

This implies that the support of $x^{\prime}$ is contained in $\{s\}$. [When $\varphi \in$ $C(S)$ is such that $\operatorname{supp}(\varphi) \subset S-\{s\}$, choose $\hat{B}(\cdot) \in C(S)$ such that $\hat{B}(s)=1$ and $\operatorname{supp}(\hat{B}(\cdot)) \subset S-\operatorname{supp}(\varphi)$. Then $\hat{B}(\cdot) \varphi(\cdot) \equiv 0$ on $S$, hence $\left\langle\varphi, x^{\prime}\right\rangle=\hat{B}(s) \cdot\left\langle\varphi, x^{\prime}\right\rangle=\left\langle\varphi, \dot{B}^{\prime} x^{\prime}\right\rangle=\left\langle B \varphi, x^{\prime}\right\rangle=\left\langle\hat{B}(\cdot) \cdot \varphi(\cdot), x^{\prime}\right\rangle=0$.] This proves the affirmation (since $x^{\prime} \neq 0$; cf. [4], p. 70).

The lemma shows that the multiplicity of the joint generalized eigenvalues of $\mathscr{A}$ with respect to the triplet $\phi \subset H \subset \phi^{\prime}$ constructed in $\S 3$ is given by

$$
\operatorname{mult}\left((\hat{A}(s))_{A \in . \mathscr{l}}\right)=\sharp\left\{i \in I: s \in S_{i}\right\} \quad(s \in S) .
$$

This formula illustrates the arbitrariness remaining in the selection of the spectral decomposition. Our construction is only well adapted to $\mathscr{A}$ with respect to the spectra.

When $H$ is separable, we can base the construction of $\phi$ on the "canonical" spectral decomposition described in $\S 2$. We then obtain:

THEOREM (4.3). (i ) $\sigma_{P}\left(\dot{B}^{\prime}\right)=\sigma\left(\dot{B}^{\prime}\right)=\sigma(B)(B \in \mathscr{B})$.

(ii) $\sigma_{P}\left(\mathscr{A}^{\prime}\right)=\sigma(\mathscr{A})$.

(iii) $\operatorname{mult}\left((A(s))_{A \in \mathscr{A}}\right)=m_{H}(s)(s \in S)$.

Proof. (i) and (ii) ensue from $S=S_{1}$, i.e., $\lambda R=S$, and the proofs of (3.1) and (3.3). (iii) is a consequence of formulas (2.2) and (4.2).

If $\mathscr{A}$ has simple spectrum (i.e., in the separable case: $\mathscr{A}$ possesses a cyclic vector, or, equivalently, $\left.m_{H}(s)=1(s \in S)\right)$ because of (4.3) (iii) the following formula holds:

$$
\operatorname{mult}\left(\left(\lambda_{A}\right)_{A \in \mathscr{A}}\right)=1 \text { for all }\left(\lambda_{A}\right)_{A \in \mathscr{A}} \in \sigma_{P}\left(\mathscr{\mathscr { C }}^{\prime}\right) \text {. }
$$


In the nonseparable case we have the following result concerning multiplicity:

Theorem (4.5). If $\mathscr{A}=\mathscr{B}$ is maximal Abelian, then (4.4) holds.

Proof. Then to $\mathscr{B}$ corresponds the full multiplication algebra $C B(R)$ on $L^{2}(R, \mu)$. As $C B(R)$ separates the points of $R=\cup_{i \in I} \widetilde{S}_{i}$, we obtain that $S_{i} \cap S_{j}=\varnothing$ for $i \neq j$. Now the affirmation ensues from (4.2).

The natural extension of the notion ". $\mathscr{A}$ possesses simple spectrum" to the nonseparable case is that the von Neumann algebra generated by $\mathscr{A}$ and 1 is maximal Abelian (cf. [19]). Theorem (4.5) says that (4.4) holds, if $\mathscr{A}$ is a von Neumann algebra with simple spectrum. We conclude by formulating a problem: Let $\mathscr{A}$ be an arbitrary system with simple spectrum. How "must" the triplet $\phi \subset H \subset \phi^{\prime}$ be constructed to obtain (4.4)?

\section{REFERENCES}

1. D.Babbit, Rigged Hilbert spaces and one particle Schroedinger operators, Rep. Math. Phys., 3 (1972), 37-42.

2. H. Baumgärtel, Resonanzen und Gelfandsche Raumtripel, Math. Nachr., 72 (1976), 93-107.

3. Yu. M. Berezanskii, Expansion in eigenfunctions of self-adjoint operators, Providence, R. I.: Amer. Math. Soc., 1968.

4. N. Bourbaki, Elements de mathématique, Livre VI. Intégration. Paris: Hermann, 1965.

5. W. G. Farris, Perturbations and nonnormalizable eigenvectors, Helvetica Phys. Acta, 44 (1971), 930-936.

6. R. Godement, Sur la théorie des représentations unitaires, Ann of Math., 53 (1951), 68-124.

7. R. A. Hirschfeld, Expansions in eigenfunctionals, Nederl. Akad. Wetensch. Proc. Ser., A 68 (1965), 512-520.

8. K. Kalb, Spectrale Invarianz bei verallgemeinerten Eigenfunktionsentwicklungen, Manuscripta Math., 7 (1972), 271-297.

9. — Die Vielfachheit nach Hellinger und die geometrische Vielfachheit der verallgemeinerten Eigenwerte, Archiv Math. (Basel), 25 (1974), 290-296.

10. Teorema y funciones de multiplicidad espectral, Rev. Col. Mat., 10 (1976), 1-37.

11. - Selbstadjungierte Operatoren als "dual rein atomare" Skalaroperatoren, Math. Ann., 232 (1978), 121-130.

12. F. Maeda, Remarks on spectra of operators on locally convex spaces, Proc. National Acad. Sci., USA, 47 (1961), 1052-1055.

13. K. Maurin, Eine Bemerkung zu allgemeinen Eigenfunktionsentwicklungen, Bull. Acad. Polon. Sci. Sér. Sci. Math. Astr. Phys., 8 (1960), 381-384.

14. General eigenfunction expansions and unitary representation of topological groups, Warszawa: PWN, 1968.

15. K. Napiórkowski, On generalized eigenfunctions of operators in a Hilbert space, 
Studia Math., 46 (1973), 79-82.

16. K. Napiórkowski, On good and bad generalized eigenvectors, $I, I I$, Bull. Acad. Polon. Sci. Sér. Sci. Math. Astr. Phys., 22 (1974), 1215-1218; 23 (1975), 251-252.

17. E. Nelson, Topics in dynamics. I: Flows, Math. Notes Princeton, Princeton: University Press, 1969.

18. Ch. E. Rickart, General Theory of Banach Algebras, Princeton: Van Nostrand, 1960. 19. I. E. Segal and R. A. Kunze, Integrals and Operators, New York: McGraw-Hill, 1968.

Received April 21, 1977.

JOHANNES GUTENBERG-UNIVERSITÄT

D6500 MAINZ

GERMANY 


\section{PACIFIC JOURNAL OF MATHEMATICS}

\section{EDITORS}

RICHARD ARENS (Managing Editor)

University of California

Los Angeles, California 90024

C. W. CURTIS

University of Oregon

Eugene, OR 97403

C. C. MOORE

University of California

Berkeley, CA 94720

\section{J. DUGUNDJI}

Department of Mathematics University of Southern California Los Angeles, California 90007

R. Finn aNd J. Milgram Stanford University Stanford, California 94305

\section{ASSOCIATE EDITORS}

E. F. BeCKenbaCH

B. H. NeumanN

F. WOLF

K. YosHIDA

\section{SUPPORTING INSTITUTIONS}

UNIVERSITY OF BRITISH COLUMBIA CALIFORNIA INSTITUTE OF TECHNOLOGY UNIVERSITY OF CALIFORNIA MONTANA STATE UNIVERSITY UNIVERSITY OF NEVADA, RENO NEW MEXICO STATE UNIVERSITY OREGON STATE UNIVERSITY UNIVERSITY OF OREGON
UNIVERSITY OF SOUTHERN CALIFORNIA STANFORD UNIVERSITY UNIVERSITY OF HAWAII UNIVERSITY OF TOKYO UNIVERSITY OF UTAH WASHINGTON STATE UNIVERSITY UNIVERSITY OF WASHINGTON 


\section{Pacific Journal of Mathematics}

\section{Vol. 76, No. $1 \quad$ November, 1978}

Ata Nuri Al-Hussaini, Potential operators and equimeasurability ......... 1

Tim Anderson and Erwin Kleinfeld, Semisimple nil algebras of type $\delta . \ldots .99$

Stephen LaVern Campbell, Linear operators for which $T^{*} T$ and $T+T^{*}$

commute. III ......................................

Robert Jay Daverman, Special approximations to embeddings of codimension one spheres...............................

Donald M. Davis, Connective coverings of $\mathrm{BO}$ and immersions of projective

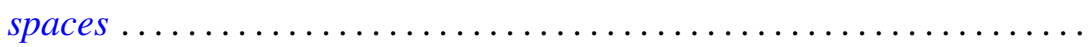

V. L. (Vagn Lundsgaard) Hansen, The homotopy type of the space of maps of

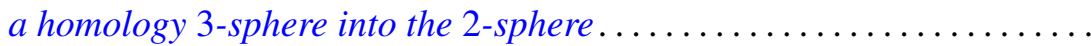

James Victor Herod, A product integral representation for the generalized

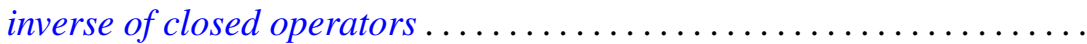

A. A. Iskander, Definability in the lattice of ring varieties ..............

Russell Allan Johnson, Existence of a strong lifting commuting with a

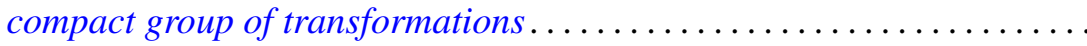

Heikki J. K. Junnila, Neighbornets...................... 83

Klaus Kalb, On the expansion in joint generalized eigenvectors . ......... 109

F. J. Martinelli, Construction of generalized normal numbers . . . . . . . . . 117

Edward O'Neill, On Massey products ....................... 123

Vern Ival Paulsen, Continuous canonical forms for matrices under unitary

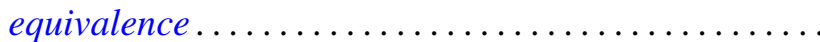

Justin Peters and Terje Sund, Automorphisms of locally compact groups . . . 143

Duane Randall, Tangent frame fields on spin manifolds . . . .

Jeffrey Brian Remmel, Realizing partial orderings by classes of co-simple sets . . . .

J. Hyam Rubinstein, One-sided Heegaard splittings of 3-manifolds ...

Donald Charles Rung, Meier type theorems for general boundary approach

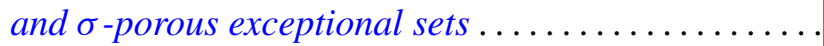

Ryōtarō Satō, Positive operators and the ergodic theorem

Ira H. Shavel, A class of algebraic surfaces of general type constructed from

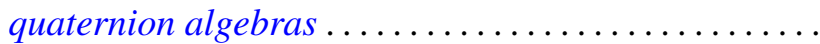

Patrick F. Smith, Decomposing modules into projectives and injectives ....

Sergio Eduardo Zarantonello, The sheaf of outer functions in the polydisc... 